\title{
More than six weeks to resolve from CT manifestations in COVID- 19 patient: A case report
}

\section{Wenxiong Xu}

Third Affiliated Hospital of Sun Yat-sen University

\section{Ziying Lei}

Third Affiliated Hospital of Sun Yat-sen University

\section{Dabiao Chen}

Third Affiliated Hospital of Sun Yat-sen University

\section{Xuejun Li}

Third Affiliated Hospital of Sun Yat-sen University

\section{Zhanlian Huang}

Third Affiliated Hospital of Sun Yat-sen University

Jing Liu

Third Affiliated Hospital of Sun Yat-sen University

\section{Zhiliang Gao}

Third Affiliated Hospital of Sun Yat-sen University

Liang Peng ( $\sim$ pliang@mail.sysu.edu.cn)

Third Affiliated Hospital of Sun Yat-sen University

\section{Case Report}

Keywords: coronavirus, COVID-19, computed tomography, lesion

Posted Date: March 31st, 2020

DOI: https://doi.org/10.21203/rs.3.rs-20188/v1

License: @ (1) This work is licensed under a Creative Commons Attribution 4.0 International License. Read Full License 


\section{Abstract}

Background: Coronavirus Disease 2019 (COVID-19) outbroke in Wuhan and spread to the world recently. But there were little studies on how long it took to recover from treatment beginning and resolve from chest computed tomography (CT) imaging so far.

Case presentation: A patient diagnosed with severe type of COVID-19 was reported in this study. He was the first patient recovered and discharged from our hospital located in Guangzhou city. Initial chest computed tomography (CT) images of him showed bilateral multiple lobular peripheral ground-glass opacities without consolidation. Features and changes of his chest CT images from admission to discharge and follow-up were demonstrated. It took more than six weeks for lesion resolution in CT manifestations although the symptoms improved for a period of time after proper treatment.

Conclusions: Repeated chest CT imaging for a period of more than six weeks in patients of COVID-19 is necessary to ascertain the lesion resolution and completely recovery. The result could be supplementary data to COVID-19 and help health care providers manage the COVID-19 patients.

\section{Background}

Coronavirus Disease 2019 (COVID-19) outbroke in Wuhan and spread to the world recently. World Health Organization reported it on January $12,2020^{[1]}$ and declared it as a global health emergency on January 30, 2020. The 2019 novel coronavirus (SARS-CoV-2) was identified by whole-genome sequencing method and described in early study by Zhu et al ${ }^{[2]}$. Huang et al ${ }^{[3]}$ demonstrated clinical features of COVID-19 patients within groups of intensive care unit (ICU) care and no ICU care. After that, supplementary data of epidemiological and clinical characteristics was demonstrated by Chen et al ${ }^{[4]}$. But there were little studies on how long it took to recover from treatment beginning and resolve from chest computed tomography (CT) imaging so far. In this study, we reported a patient diagnosed with severe type of COVID-19 from admission to discharge and follow-up. Clinical characteristics, including chest CT manifestations, were demonstrated.

\section{Case Presentation}

A 37-year-old man presented a 4-day history of fever and cough before admission. The highest temperature was $38.3^{\circ} \mathrm{C}$. He was isolated and treated in isolation unit, Department of Infectious Diseases of Third Affiliated Hospital of Sun Yat-sen University from January $22^{\text {nd }}, 2020$ to February $7^{\text {th }}, 2020$. He had exposure history to suspected patient who travelled back from Wuhan, China. He denied of exposure to the Huanan seafood market or wild animals. His wife was confirmed diagnosed with COVID-19 two days after his admission. He was a non-smoker without any comorbidity.

At admission, the patient's body temperature was $37.3^{\circ} \mathrm{C}$, blood pressure was $131 / 65 \mathrm{~mm} \mathrm{Hg}$, pulse was 104 beats per minute, respiratory rate was 18 breaths per minute, and pulse oxygen saturation $\left(\mathrm{SpO}_{2}\right)$ is $98 \%$ while he was breathing ambient air. Auscultation of lungs showed no wet or dry rales. Arterial blood gas analysis indicated no abnormality with arterial partial pressure of oxygen ( $\mathrm{PaO2}$ ) of $94.1 \mathrm{~mm} \mathrm{Hg}$ and Oxygenation Index (arterial partial pressure of oxygen to fraction of inspiration oxygen $\left[\mathrm{PaO}_{2} / \mathrm{FiO}_{2}\right]$ ) of $448 \mathrm{~mm} \mathrm{Hg}$. Other laboratory examinations showed normal blood cell count, normal level of biochemical tests (hepatic function, renal function, cardiac function and coagulation function), normal level of procalcitonin, C-reactive protein and erythrocyte sedimentation rate. Antigen tests for Influenza A, B and IgM tests for Influenza A, B, Parainfluenza, Respiratory Syncytial Virus, Adenovirus, Mycoplasma Pneumoniae, Chlamydia Pneumoniae, Rickettsia Burneti, Legionella Pneumophila were all negative. Blood cultures for bacterium (aerobic and anaerobic) and fungus were negative. Detection of SARS-CoV-2 nucleic acid from his oropharyngeal swab was positive by Center for Disease Control and Prevention (CDC) of Guangzhou city. Unenhanced chest CT images showed bilateral multiple lobular peripheral ground-glass opacities (GGO) without consolidation (Figure 1A). Diagnosis with COVID-19 was confirmed.

After admission, the patient received antiviral therapy of oral arbidol, oral lopinavir and ritonavir, and interferon alfa-2b inhalation. Antibiotic agent of Moxifloxacin, human immunoglobulin injection and nasal cannula oxygen support were given to him. The symptoms did not improve until day $8^{\text {th }}$ after admission. He was diagnosed with severe type of COVID-19 as his arterial blood gas analysis indicated abnormality with $\mathrm{PaO} 2$ of $79.9 \mathrm{~mm} \mathrm{Hg}$ and Oxygenation Index of $242 \mathrm{~mm} \mathrm{Hg}$ on day $9^{\text {th }}$ after admission, according to diagnosis and treatment program in our country ${ }^{[5]}$. Repeated chest CT imaging were finished in the course to ascertain the change of manifestations, which demonstrated new GGO on day $3^{\text {rd }}$ (Figure 1B), progression on day $11^{\text {th }}$ (Figure 1C) and partial absorption of the lesion on day $14^{\text {th }}$ (Figure 1D) after admission. Two consecutive negative results of detection the SARS-CoV-2 nucleic acid from his 
oropharyngeal swabs by CDC on day $13^{\text {th }}$ and day $16^{\text {th }}$ after admission, with an interval of 72 hours, were obtained before he was discharged on day $17^{\text {th }}$ after admission (Table 1). The treatment mentioned above was given to him until he was discharged. After discharge, he did not receive any drug as he felt normal.

Table 1 Detection of nucleic acid and antibody of SARS-CoV-2

\begin{tabular}{|lllllll|}
\hline & $1-23$ & $1-29$ & $2-3$ & $2-6$ & $3-4$ & $3-18$ \\
\hline Nucleic acid & + & + & - & - & - & $/$ \\
\hline $\lg M$ & $/$ & $/$ & $/$ & $/$ & - & - \\
\hline $\lg G$ & $/$ & $/$ & $/$ & $/$ & + & + \\
\hline
\end{tabular}

For further examinations in the follow-up, the patient came back to our hospital without any symptoms on March $4^{\text {th }}, 2020$ (day $26^{\text {th }}$ after discharge). SARS-CoV-2 nucleic acid from his oropharyngeal swabs and serum SARS-CoV-2 IgM were negative, serum SARSCoV-2 IgG was positive (Table 1). Chest CT images showed that most of the lesions were resolved, with some remaining patchy opacities in bilateral lower lobes (Figure 1E). After that, serum SARS-CoV-2 IgM was negative and IgG was positive on March $18^{\text {th }}, 2020$ (day $40^{\text {th }}$ after discharge) (Table 1 ).

\section{Discussion And Conclusions}

SARS-CoV-2 was similar to Severe Acute Respiratory Syndrome Coronavirus (SARS-CoV) which was globally spread from November 2002 to July $2003^{[6]}$, and Middle East Respiratory Syndrome Coronavirus (MERS-CoV)which was first reported in Saudi Arabia in 2012 [7]. COVID-19 ranged from mild type to life-threatening. Acute respiratory distress syndrome (ARDS) and/or multiple organ disfunction syndrome (MODS) were recorded ${ }^{[3,4]}$. This reminds us not to underestimate the severity of the disease. It is still unknown how long it takes COVID-19 patients from symptoms onset to recovery, or from admission to hospital, receiving proper treatment, to discharge from hospital.

Initial chest CT images of the patient in our case report demonstrated that GGO were found without specificity. Similar findings were reported in two studies ${ }^{[8,9]}$. New GGO in right upper lobe, right lower lobe and left lower lobe were visible on day $3^{\text {rd }}$ after admission although he was receiving proper treatment. This finding is similar to the first case of COVID-19 in the United States ${ }^{[10]}$, who took more than 11 days of treatment in hospital, with remaining cough unresolved, increasing left basilar opacity was visible on day $5^{\text {th }}$ and stable streaky opacities in the lung bases were visible on day $6^{\text {th }}$ after his admission. In our case report, the symptoms of the patient did not improve until day $8^{\text {th }}$ after admission. Only partial absorption without absolutely resolution of the opacities was visible on day $14^{\text {th }}$ after admission before he was discharged, although the symptoms improved for a period of time. He had remaining patchy opacities in bilateral lower lobes in chest CT images on day $26^{\text {th }}$ after his discharge. It seemed to take a long period of time for COVID-19 patients from treatment beginning to recovery, and for complete lesion absorption in chest CT imaging.

In Huang's study, fitness for discharge was based on abatement of fever for at least 10 days, with improvement of chest radiographic evidence and viral clearance in respiratory samples from upper respiratory tract ${ }^{[3]}$. Normal body temperature maintaining for 3 days, obvious improvement of respiratory symptoms, absorption of inflammation in chest radiographic manifestation and two negative results of detecting SARS-CoV-2 nucleic acid from respiratory samples with an interval of more than 24 hours, are all recommended for patients discharging from hospital in diagnosis and treatment program in our country ${ }^{[5]}$. All the discharged patients are also recommended to take home quarantine and self-health monitoring for at least 14 days.

Our case report indicated that repeated chest CT imaging for a period of more than six weeks in COVID-19 patients is necessary to ascertain the lesion resolution and complete recovery. The result could be supplementary data to COVID-19 and help health care providers manage the COVID-19 patients.

\section{List Of Abbreviations}

COVID-19: Coronavirus Disease 2019 
SARS-CoV-2: the 2019 novel coronavirus

ICU: intensive care unit

CT: computed tomography

$\mathrm{SpO}_{2}$ : pulse oxygen saturation

$\mathrm{PaO}_{2}$ : arterial partial pressure of oxygen

$\mathrm{FiO}_{2}$ : fraction of inspiration oxygen

CDC: Center for Disease Control and Prevention

GGO: ground-glass opacities

SARS-CoV: Severe Acute Respiratory Syndrome Coronavirus

MERS-CoV: Middle East Respiratory Syndrome Coronavirus

ARDS: acute respiratory distress syndrome

MODS: multiple organ disfunction syndrome

IRB: institutional review board

\section{Declarations}

\section{Acknowledgments}

We thank all the medical staff of the isolation unit of Third Affiliated Hospital of Sun Yat-sen University.

\section{Author Contributions}

Liang Peng and Wenxiong Xu had full access to all of the data in the study and takes responsibility for the integrity of the data and the accuracy of the data analysis.

Concept and design: All authors.

Acquisition, analysis, or interpretation of data: Wenxiong Xu.

Drafting of the manuscript: Wenxiong $\mathrm{Xu}$.

Obtained funding: Liang Peng and Zhiliang Gao.

Supervision: Liang Peng and Zhiliang Gao.

\section{Ethic approval}

This study was approved by the institutional review board (IRB) of Third Affiliated Hospital of Sun Yat-sen University. All patients voluntarily signed an informed consent form approved by the IRB before participation.

\section{Funding}

This study was supported by grants from Natural Science Foundation of China (NSFC) (grant number 81570539 and 81873572 ), Tackling of key scientific and emergency special program of Sun Yat-sen University (SYSU-TKSESP) (without grant number) and Emergency special program for 2019-nCoV of Guangdong province science and technology project (GDSTP-ESP) (grant number 2020B111105001). 
Role of the Funder. The NSFC, SYSU-TKSESP and GDSTP-ESP had no role in the design and conduct of the study; collection, management, analysis, and interpretation of the data; preparation, review, or approval of the manuscript; and decision to submit the manuscript for publication.

\section{Declaration of interests}

All authors declare no competing interests.

\section{References}

[1] World Health Organization. Novel coronavirus - China. January 12, 2020. http://www.who.int/csr/don/12-january-2020-novelcoronavirus-china/en/ (accessed February 13, 2020)

[2] Na Zhu, Dingyu Zhang, Wenling Wang, et al. A Novel Coronavirus from Patients with Pneumonia in China, 2019. New England Journal of Medicine. 2020. https://doi.org/10.1056/NEJMoa2001017

[3] Chaolin Huang, Yeming Wang, Xingwang Li, et al. Clinical features of patients infected with 2019 novel coronavirus in Wuhan, China. Lancet. 2020. https://doi.org/10.1016/S0140-6736(20)30183-5.

[4] Nanshan Chen, Min Zhou, Xuan Dong, et al. Epidemiological and clinical characteristics of 99 cases of 2019 novel coronavirus pneumonia in Wuhan, China: a descriptive study. Lancet. 2020; 395: 507-513. https://doi.org/10.1016/S0140-6736(20)30211-7

[5] National Health Commission of the People's Republic of China. Diagnosis and treatment program for 2019 Novel Coronavirus pneumonia. January 22, 2020.

http://www.nhc.gov.cn/xcs/zhengcwj/202001/f492c9153ea9437bb587ce2ffcbee1fa/files/39e7578d85964dbe81117736dd789d8f.pdf (accessed February 13, 2020)

[6] World Health Organization. Summary of probable SARS cases with onset of illness from 1 November 2002 to 31 July 2003. December 31, 2003. https://www.who.int/csr/sars/country/table2004_04_21/en/ (accessed February 13, 2020)

[7] World Health Organization. Middle East respiratory syndrome coronavirus (MERS-CoV). November, 2019. http://www.who.int/emergencies/mers-cov/en/ (accessed February 13, 2020)

[8] Michael Chung, Adam Bernheim, Xueyan Mei, et al. CT Imaging Features of 2019 Novel Coronavirus (2019-nCoV). Radiology. 2020. https://doi.org/10.1148/radiol.2020200230

[9] Fengxiang Song, Nannan Shi, Fei Shan, et al. Emerging Coronavirus 2019-nCoV Pneumonia. Radiology. 2020. https://doi.org/10.1148/radiol.2020200274

[10] Michelle L. Holshue, Chas DeBolt, Scott Lindquist, et al. First case of 2019 Novel Coronavirus in the United States. The New England Journal of medicine. 2020. https://doi.org/10.1056/NEJMoa2001191

\section{Figures}


A

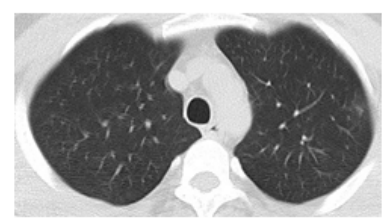

B

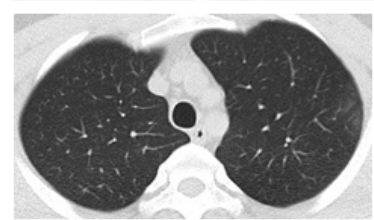

$\mathrm{C}$

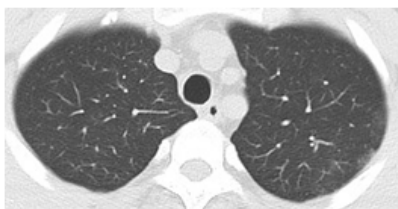

$\mathrm{D}$

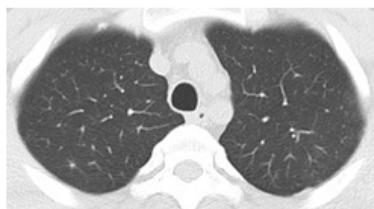

$\mathrm{E}$

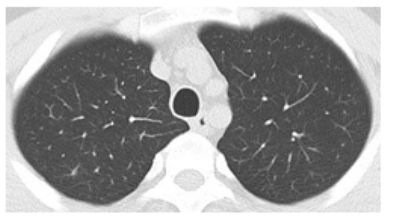

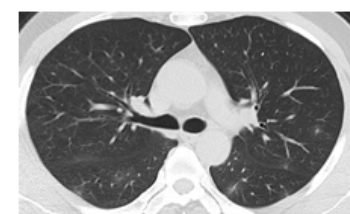
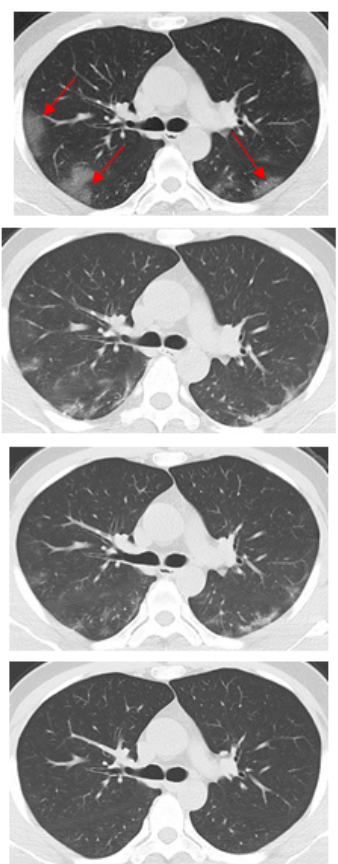
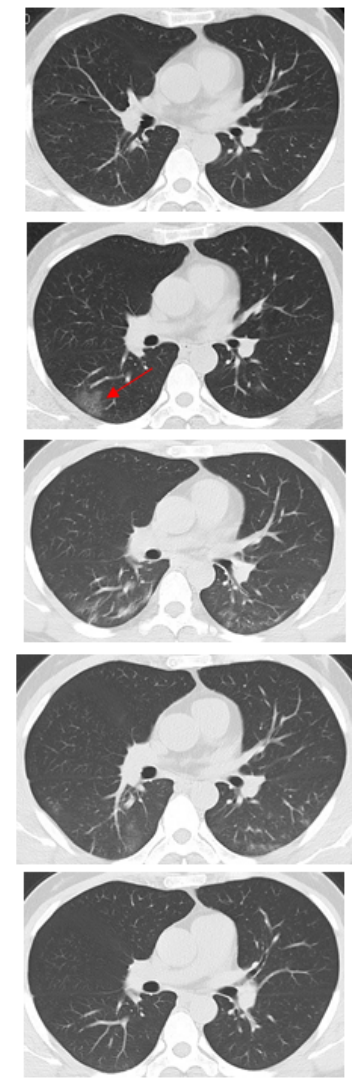
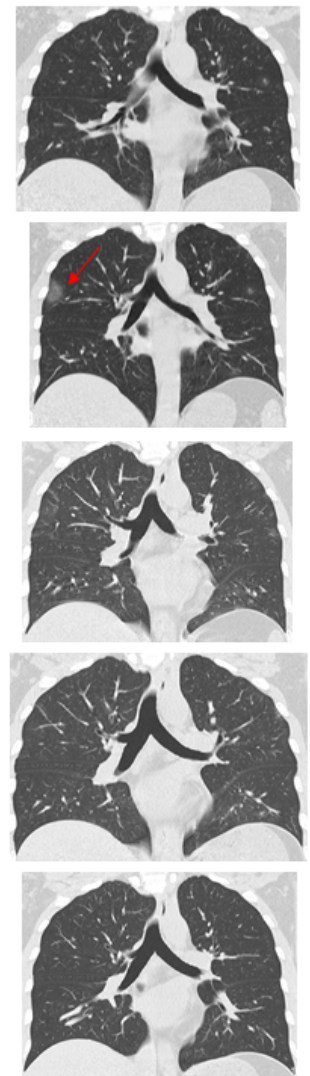

\section{Figure 1}

Chest CT images from admission to discharge and follow-up. (A) Bilateral multiple lobular ground-glass opacities without consolidation were visible at admission. (B) New ground-glass opacities in right upper lobe, right lower lobe and left lower lobe were visible on day 3rd after admission (red arrow). (C) Progression of the lesions was visible on day 11th after admission. (D) Partial absorption of the lesions was visible on day 14th after admission. (E) Resolution of most of the lesions with some remaining patchy opacities in bilateral lower lobes were visible on day 26th after discharge. 